\title{
Research on Computer Assisted Translation Technology Teaching Model
}

\author{
Min Luo \\ School of Foreign Languages, Wuhan Polytechnic University, Wuhan, 430028, China
}

Keywords: CAT; Translation teaching; Teaching model.

\begin{abstract}
As international trade has become increasingly prosperous, and cultural exchanges has become more and more frequent, translation work has met with higher and higher requirements. As a newly emerged technology, CAT has become a great driving force to translation work by facilitating the work of foreign language workers. Therefore, setting up computer-assisted translation courses for college students is a must-go move that conforms to the trend of the times. It is promising to introduce CAT as a translation and teaching tool to classes by combining computers with translation teaching well.
\end{abstract}

\section{Introduction}

In the past 20 years, the improvement of political and economic policies in various countries in the world, the increase of trade exchanges, and the integration of cultural practices have made the demand for translation between different languages increasingly explosive. With the advent of a high-tech era, the improvement of information technology has provided strong technical support for computational linguistics, and has spawned a variety of computer translation tools, such as online translation, electronic dictionaries, machine translation software (MT), corpus search tools, etc. We begin to notice them in our lives and begin to get used to using them. Its full practicality has prompted colleges and universities to offer related courses and majors. Its superiority compared to traditional translation and its current development status have reminded us that we should increase our research on this new discipline.

This article is based on practicality, focusing on the combination of translation practice, translation skills and CAT technology, to explore the translation teaching model of CAT technology in undergraduate period. We aim to improve students' translation ability and translation efficiency so as to enhance their employment response ability. By mastering the use of CAT technology, students will be fully equipped for applying for part-time translation work. The economic benefits and market recognition brought by learning will further stimulate students' passion for learning.

\section{Some Basic Concepts of CAT}

CAT (computer-assisted translation) is the development of machine translation or computer translation. It refers to the automatic translation of computer programs in the process of human translation. Computer programs will match a word and its translation by retrieving directly from its translation memory, which greatly reduces the workload of translators. People often confuse our translation tools, such as Google and Weidao, which we often use in translation, with computer-assisted translation. However, the translation tools above belong to the category of machine translation. The biggest feature of CAT is that it does not rely on the automatic translation of machines, but is a process that people participate, which is the improvement and extension of machine translation. The quality of computer translation is equal to or better than that of human translation, but it is less time-consuming and can yield twice the result with half the effort. It has unparalleled superiority and can be a "shortcut" in the translation process.

Generally speaking, CAT technology can be explained in both broad and narrow sense. "Broadly speaking, it refers to a computer tool that can assist translators in translation, such as word processing software, electronic dictionaries, corpus, search engines, CAT software, etc."; and in a narrow sense, it refer to "computer-aided translation software specially designed to improve translation efficiency and optimize the translation process, including translation memory tools, terminology management tools, 
project management tools, etc. "The object of this study is the former, which is a computer tool that can help with translation, including computer-assisted translation software.

\section{The Feasibility of Implementing CAT Teaching at Undergraduate Level}

As the main force in training English translators, undergraduate translation teaching does not follow the development of CAT technology, and CAT courses cannot be popularized in the undergraduate stage. However, by thinking deeply the reasons behind, we find that these problems can be effectively resolved. The following will explain the conditions for the using of CAT technology in the undergraduate stage from three aspects: motivation, equipment, and teachers.

Strong teaching motivation.In the age of information, traditional manual translation is hard to meet the needs of modern translation. Social requirements for professional translation generally include the ability to use tools, that is, to master various auxiliary translation tools expertly. Only with the master of certain translation techniques, it is possible for undergraduates who are the main translators to integrate smoothly into the work flow of a translation company. The strong market demand has spawned the needs of students, and the students' motivation for learning has provided the impetus for undergraduate CAT technology teaching.

Free CAT resources. For undergraduate CAT teaching, the problem of insufficient software equipment is one of the reasons that restrict the opening of CAT technology courses. Mainstream CAT software includes SDL Trados, DéjàVu, Wordfast, etc. The standalone editions of these software are expensive, and the standalone version of CAT software has a market price of several thousand yuan or even tens of thousands. Therefore, undergraduate CAT teaching faces such contradictions. On the one hand, teaching concepts are lagging behind, and equipment is in short supply; on the other hand, market demand is strong and learning motivation is high. To ease this conflict, we can consider using free CAT resources. For example, according to the introduction of Zhou Xinghua, " Snowman CAT is a kind of domestic CAT software, and the stand-alone free version system of it comes with 300,000 English-Chinese and Chinese-English dictionaries. Although the function is limited, it can also meet the general translation needs and can be used in actual translation work and it can be deemed as an introduction to CAT." In addition, many free website resources, such as Answer4cat, TransGas, etc., can provide free CAT resources. Expensive CAT software should not become a stumbling block to CAT technology teaching, and the free Internet CAT resource opens the door to CAT teaching.

Multi-channel teacher training. In the current CAT teaching, teacher shortage is always a disturbing topic. However, at present, the development of CAT is gratifying. Numerous free Internet CAT resources and teaching videos, translation technology seminars or training courses organized by associations or institutions, and MOOC courses related to computer-aided translation teaching at Peking University provide multiple channels for teacher training. Besides, CAT teaching in undergraduate courses can mainly focus on practice, and the theoretical part can be explained briefly. As long as there are certain computer-based translation teachers, they can be qualified for undergraduate CAT teaching after a period of training, because undergraduate CAT teaching only requires students to get started. Students can continue to learn more and in-depth about CAT technology by conducting self-learning, advanced studies, etc. Multi-channel teacher training solves the problem of CAT teaching in undergraduate education.

\section{CAT Teaching Model Design}

CAT teaching content. The teaching content of CAT technology must be organically integrated into the undergraduate traditional translation teaching. The content can be divided into different modules and be flexibly inserted into the traditional translation teaching content. The core teaching content of CAT technology can be divided into the following three modules.

\section{Translation Information Technology}

This article discusses the translation information technology module, mainly including the search technology, and the digital text acquisition technology. Search techniques include the use of various web 
search engines, academic databases, corpus, electronic dictionaries, electronic encyclopedias and other assisted translation tools. Mastering some advanced search techniques can usually solve the problems that cannot be handled by using common keyword search and improve the efficiency and the quality of translation. In addition, CAT software deals with electronic documents, but in actual translation work, customers only provide source documents in paper prints. To convert the paper version of a document to an electronic one, you must learn to use optical character recognition (OCR) technology. The digital text acquisition technology mainly introduces commonly used OCR software.

\section{Software Application Technology}

CAT software application technology is a core part of CAT technology that translators should master. In this module, we will pay attention to the introduction of free "snowman" CAT software. CAT software application technology teaching should include the basic knowledge of CAT software, software installation, quick start, introduction of commonly used functions of the software, the translation demonstration of various file formats, the integration of network resources, memory bank creation, and memory bank search, etc.

\section{Translation Project Management Content}

In the translation market, translation is a complete industrial process, including receiving tasks, project analysis, project management, collaborative translation, etc. It cannot be limited to the language conversion process from the source language text to the target language text. Therefore, the entire simulation of a translation process should be part of teaching. This part of the teaching content is mainly the creation of a network collaborative translation platform, including shared memory database, terminology database, synchronization of review and translation, collaborative translation of project document, and quality control.

CAT Teaching Assessment Model. Mainly adopting a combination of formative assessment and summative assessment, CAT teaching is a multiple evaluation system focusing on formative assessment. The formative assessment mainly includes the conditions of students' pre-learning and task completion. The CAT software teaching adopts the form of a flipping classroom, and students are supposed to complete the video learning and some simple tasks related to the video content. Each student group has to do translation report, that is, as a translation project comes to an end, each group separately describes the preparation work for the project implementation, the mid-term practice and the summary of the project, and each group will make the report from three dimensions: translation, techniques, and team division of labor. By doing this, better understanding and communication among groups about the process and experience of each other's translation project will be promoted. Therefore, students' translation skills, CAT software application capabilities, and team collaboration capabilities will be trained and enhanced. Translation quality assessment, the team learning files of a translation project will be established to register the quality of each completed project, working as one of the basis for formative evaluation. In addition, the final test score is the main basis for examining students' bilingual translation ability and is the main component of summative assessment. Since CAT technology is organically integrated into traditional translation courses, the technical teaching of the course mainly adopts formative evaluation methods, and the final test of bilingual translation ability is the main component of summative evaluation of students.

\section{A Reflection on CAT Teaching}

Computer-assisted translation (CAI), as a new curriculum with a short history of development, is obviously a weak link in our teaching. Therefore, we should actively learn from schools with rich experience in computer-assisted translation studies to make up for our deficiencies.

First, as an emerging high-tech translation method, equipment and systems are indispensable. Therefore, the first schools or institutions that offer such courses should provide hardware equipment as much as possible to provide strong protection for practice. After all, the computer-assisted translation technology cannot be really mastered by only studying the theory. We could also make effective use of network information resources. There are relatively adequate introductions about the basic concept of 
computer translation theory and the download and operation method of specific software on the network. Teachers and students can fully utilize network resources in learning.

Second, teachers' own knowledge reserve and practical operation ability. As a main subject of science, computer can pose a great challenge on teachers of foreign languages in its combination with translation. As a teacher of computer translation, the teacher not only needs solid language skills, but also is supposed to understand machine translation, computer translation history, and other theoretical knowledge. The teacher also has to master the specific operation of the software. In schools that open computer translation course earlier, teachers would be provided with some training courses designed for them. Also, teachers can get to know the specific operation method through network. Anyway, teachers are required to put a large amount of time and energy in learning the techniques, and in the process of it, teachers will learn to enrich their teaching and make their translation work faster and easier.

Third, the selection of teaching materials and original texts for lectures. The translation work involves a wide range of uses and different purposes. Students should be allowed to adapt to different types of texts during computer-assisted translation. The greatest advantage of computer-assisted translation is that there are different professional vocabularies and memory functions based on "translation memories." As a result, students can experiment with different texts such as texts of political society, financial trade, and technology to master the skills and techniques of different text translations and to adapt to translation work in advance.

\section{Summary}

This paper explores the CAT teaching model for undergraduate English majors. The goal is not to study advanced CAT principles and software, but to be practical and to focus on the combination of translation practice, translation capabilities, and CAT technology. It is not advisable to make the content of the translation technology too complicated for undergraduate students to learn, as long as the content can meet the requirements of later projects. Teachers should encourage students to use different CAT software through various channels to broaden their horizons. On the surface, the study of the CAT technology has a high requirement on students' computer skills, but actually, students only need to have a certain degree of computer knowledge and application ability.

\section{References}

[1]Aysha Abughazzi.Translation and Public Opinion:The Press in Jordan[C]. Ottawa:University of Ottawa (Canada),2006:106-117;278.

[2]Liu Lan. Reflections on Cultivating International Innovative Foreign Language Talents - Cultivation of Translators' Localization Awareness [J]. Science \& Technology Vision, 2014, (23).

[3]Zhang Qian . The application of computer-aid translation [J] . Journal of Jixi University(comprehensive edition), 2012, (6).

[4]Li Jiajun, Zhong Lanfeng. Illustration Principles for Parallel Corpus-based Active Chinese-English Dictionary Compilation [J]. Journal of Jiangsu University(social science edition), 2011, (2).

[5]Huang Lipo, Zhu Zhiyu. The establishment of English-Chinese corpus in China and current situation and outlook of the research $[\mathrm{J}]$. Contemporary Foreign Languages Studies, 2013，(1). 